\title{
Influence of descending height of standardized antagonist for the in-vitro wear resistance of dental composite materials
}

\author{
Ivan Chakalov' ${ }^{1}$, Pavlina Koleva ${ }^{2}$, Ivan \\ Gerzhikov $^{1}$, Nickolay Apostolov ${ }^{1}$
}

1. Department of Prosthetic Dentistry, Faculty of Dental Medicine, Medical University - Sofia, Bulgaria;

2. Dentist with a Private Practice - Sofia, Bulgaria;

\begin{abstract}
The laboratory variables under which an in vitro study is run can greatly influence the experimental results. For the in-vitro test to determine dental materials wear resistance numerous factors such as: force, contact time, wear mechanism implicated, sliding distance, surface rougness, antagonistic material and standardization, were reported to influence the experimental result. The purpose of the present paper is to emphasize the role of another factor- the height of rise and descent of the antagonist - a factor that is not mentioned when the setting of the chewing simulation device are discussed. The chewing simulator "Sofia" was used for the study. Two groups of specimens from a dental composite material were prepared following the protocol and tested under identical conditions with the only difference of different descending height $-0,1 \mathrm{~mm}$ and $0,5 \mathrm{~mm}$ respectively. Within the limits of our study a significant difference in the results was demonstrated thus proving the importance of the descending height as a factor influencing the in-vitro tests of dental material wear resistance.
\end{abstract}

Keywords: descent height, in-vitro tests, chewing simulator, wear resistance, laboratory variables 


\section{Introduction}

The laboratory variables under which an in vitro study is run can greatly influence the experimental results. For the in-vitro test to determine dental materials wear resistance numerous factors such as: loading force, contact time, wear mechanism implicated, artificial food medium, sliding distance, surface roughness, antagonistic material, were reported to influence the experimental result. As far as the authors are informed one condition has never been taken into consideration namely the height of rise and descend of the antagonist imitating chewing process. We noticed that the slight variations in rising/descending height leads to significant differences in the outcomes. The chewing simulator "Sofia" used for the in vitro wear tests was described in a previous paper (1).

\section{Aim}

The aim of the present study is to define and stress the importance of the descent height during in-vitro wear tests over the wear resistance results.

\section{Materials and methods}

\section{Composite material samples fabrication}

Four specimens were fabricated from composite material Herculite XRV. For this purpose metal rings with an outer diameter of $25 \mathrm{~mm}$, inner diameter $24 \mathrm{~mm}$ and $8 \mathrm{~mm}$ height were used. The metal rings were positioned in a specially designed holder, which was firmly fixed onto a glass slab with a smooth surface. 22.5 grams of the tested material was placed into the ring and carefully pressed against the glass slab until a uniform layer of about $1-2 \mathrm{~mm}$ was created. The polymerization of the material was done with a $2000 \mathrm{~mW} / \mathrm{cm} 2$ light-curing lamp. The surface of the samples was divided in 9 areas (with a size roughly coinciding to that of the light-transmitting tip of the lamp.) The polymerization was done separately for each area using two polymerization modes - first with the "soft start" mode for 20 seconds (with low light intensity at the beginning and a gradual increase of intensity towards the end of the curing cycle) and then with the pulse mode (with light impulses at a maximum intensity followed by a period of lack of light irradiation). Subsequently the remaining volume of the sample was filled up with pink cold-curing resin (Duracryl, Spofa Dental).

\section{Chewing simulator settings}

The settings of the chewing simulator Sofia were as follows:

- dynamic loading force: 0-50 N

- frequency $1 \mathrm{~Hz}$

- number of specimens tested simultaneously 4

- length of sliding track $7 \mathrm{~mm}$

- number of testing cycles 100000

- experimental medium- distilled water 
- antagonistic material - silicon nitride ceramic bearings pearls

- descending height - 0,1 for group 1; 0,5 for group 5

The specimens were divided into 2 groups of 2 specimens. The only factor that defers between the two groups was the descent height $-0,1 \mathrm{~mm}$ for the first group and $0,5 \mathrm{~mm}$ for the second. Apart from the descent height all the other parameters of the tests were identical for the two groups. All tests were run in distilled water in order to minimize the variations in the outcomes.

\section{Statistics}

The data was recorded and processed with the statistical package IBM SPSS Statistics 22.0.

$P<0,05$ was set as the significance level at which the zero hypothesis would be rejected. The following methods were applied:

1. Descriptive analysis

2. Analysis of variance

3. Nonparametric Kolmogorov-Smirnov test and Shapiro-Wilk test

4. One-way ANOVA and Bonferroni post hoc test

5. Student's t-test

\section{Results}

Table 1 and 2 depict the variational analysis of the wear rate by descending height $0,1 \mathrm{~mm}$ and $0,5 \mathrm{~mm}$ respectively.

Table 1: Variational analysis of the wear rate by $0,1 \mathrm{~mm}$ descending height

\begin{tabular}{|c|c|c|c|c|c|c|c|}
\hline $\begin{array}{c}\mathrm{Y} \\
\text { Mm of sliding path }\end{array}$ & Sample & $\mathrm{N}$ & $\bar{X}$ & & Min & $\mathrm{Ma}$ & \\
\hline 1 & $1-2$ & 10 & $56,40^{a}$ & 9,35 & 42,00 & 67,00 & 16,58 \\
\hline 2 & $1-2$ & 10 & $75,50^{\mathrm{b}}$ & 9,73 & 58,50 & 87,50 & 12,89 \\
\hline 3 & $1-2$ & 10 & $94,55^{\mathrm{c}}$ & 10,84 & 73,00 & 111,00 & 11,46 \\
\hline 4 & $1-2$ & 10 & $112,10^{\mathrm{d}}$ & 13,14 & 88,50 & 133,50 & 11,72 \\
\hline 5 & $1-2$ & 10 & $130,65^{\mathrm{e}}$ & 13,87 & 107,50 & 156,00 & 10,62 \\
\hline 6 & $1-2$ & 10 & $148,50^{f}$ & 12,61 & 124,00 & 166,50 & 8,49 \\
\hline
\end{tabular}

* different letters indicate significant difference $(p<0,05)$ 
Table 2: Variational analysis of the wear rate by $0,5 \mathrm{~mm}$ descending height

\begin{tabular}{|c|c|c|c|c|c|c|c|}
\hline Y & Sample & $\mathrm{N}$ & $\overline{\mathrm{X}}$ & SD & Min & Max & V \\
\hline 1 & $3-4$ & 10 & $83,75^{a}$ & 18,70 & 57,00 & 108,00 & 22,33 \\
\hline 2 & $3-4$ & 10 & $101,45^{a c}$ & 20,68 & 69,50 & 125,50 & 20,38 \\
\hline 3 & $3-4$ & 10 & $115,75^{\mathrm{bc}}$ & 21,94 & 79,50 & 143,50 & 18,95 \\
\hline 4 & $3-4$ & 10 & $126,60^{\mathrm{bc}}$ & 22,16 & 87,00 & 157,00 & 17,50 \\
\hline 5 & $3-4$ & 10 & $138,85^{\mathrm{b}}$ & 26,77 & 96,00 & 186,00 & 19,28 \\
\hline 6 & $3-4$ & 10 & $144,25^{b}$ & 22,75 & 105,50 & 178,00 & 15,77 \\
\hline
\end{tabular}

* same letter after results indicate no significant difference, different letters indicate significant difference $(p<0,05)$

For both descending heights $(0,1 \mathrm{~mm}$ for samples 1 and 2 and 0,5mm for samples 3 and 4$)$ the lowest wear rate are recorded by $y=1$ and greatest - by $y=6$. The opposite results show the variations - the highest variations are documented by $\mathrm{y}=1$ and the lowest - by $\mathrm{y}=6$.

Table 3 compares the wear rate by descending height $0,1 \mathrm{~mm}$ and $0,5 \mathrm{~mm}$ and following conclusions are to be drawn:

- The greater descending height $(0,5 \mathrm{~mm})$ leads to greater wear as the average wear depth is 118 while by descending height of $0,1 \mathrm{~mm}$ the average depth is 103 .

- The Student's t-test proves a significant difference between the wear rates by the two descending heights $(p=0,009)$.

Table 3: Comparison between the wear rates by descending height 0,1mm (Sample 1 and 2) and 0,5mm (Samples 3 and 4)Table 3: Comparison between the wear rates by descending height $0,1 \mathrm{~mm}$ (Sample 1 and 2) and 0,5mm (Samples 3 and 4)

\begin{tabular}{lcccccc}
\hline Descending height & Sample & $\mathrm{Y}$ & $\begin{array}{c}\text { Number of } \\
\text { experiments }\end{array}$ & $\overline{\mathrm{X}}$ & $\mathrm{SD}$ & $\mathrm{V}$ \\
\hline $0,1 \mathrm{~mm}$ & $1-2$ & $1-6$ & 60 & $102,95^{\mathrm{a}}$ & 33,59 & 32,63 \\
$0,5 \mathrm{~mm}$ & $3-4$ & $1-6$ & 60 & $118,44^{\mathrm{b}}$ & 30,07 & 25,39 \\
\hline
\end{tabular}

* different letters indicate significant difference $p=0,009$

\section{Discussion}

The chewing simulator "Sofia" utilized in the present study was constructed in order to reproduce the dynamic force profile during physiological chewing process identical to in vivo data (2), as well as in 
accordance with the occlusal theory of Le Gall and Lauret (3). While classic occlusion theory is mostly concerned about centrifugal movements, the physiological occlusion theory reminds us that human beings chew mostly in inward-oriented chewing cycles (centripetal movement) (Fig.1) with a subsequent distinct differences in occlusal contacts and loadings. Among the most specific features of the chewing simulator "Sofia" is the ability to reproduce contacts and force profile loading during the physiological centripetal movement (Fig. 2). The chewing cycle as simulated by the device consists in four phases:

1. descending of the antagonist towards the specimen (this movement reproduces the initial contact between the buccal cusps of the antagonist teeth during the chewing cycle)

2. sliding motion between the antagonist and the specimen under variable load determined in advance (this corresponds to the sliding motion from the point of initial contact to the position of central occlusion which is accompanied by an increase in load until it reaches its maximum value; the subsequent sliding movement during the cycle out is carried out under load lower than the maximum)

3. separation of the antagonist from the specimen (it reproduces the end of the occlusal phase of the chewing cycle)

4. return of the system in a starting position (preparation of the dentition for a new occlusal phase)
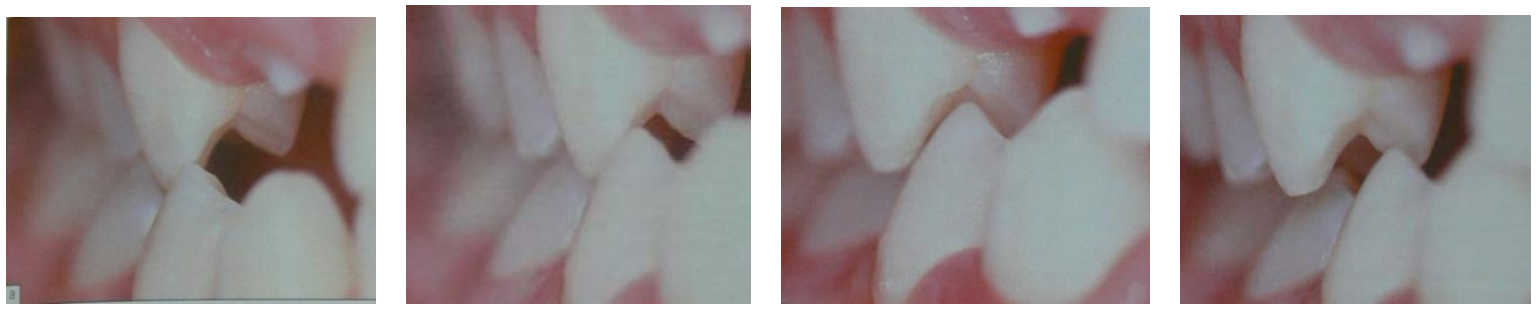

Fig 1. Presentation of the occlusal stages of one chewing cycle (according to Le Gall and Lauret)

(1)

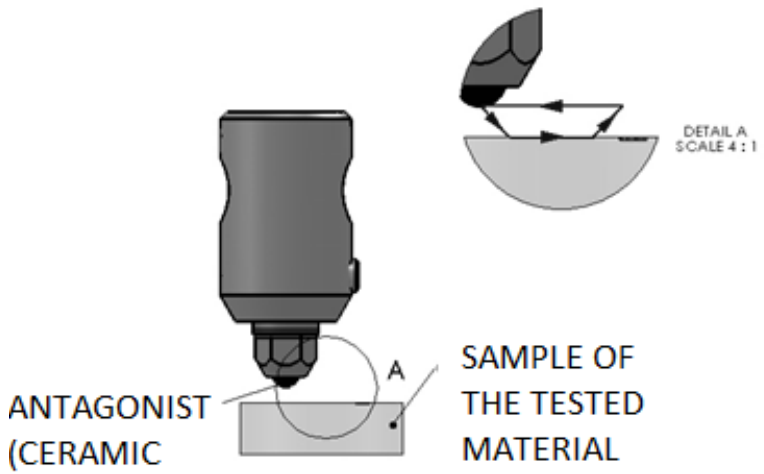

PEARL)

Fig.2 The antagonist trajectory towards the sample during one chewing cycle 
Regarding the best artificial food medium for in-vitro tests on mastication and wear, it is still a matter of discussion in literature. Experiments have been carried out with flour, poppy seeds, millet and rice seeds, PMMA, silicon carbide or calcium carbonate particles. In this experiment we aimed to exclude the possible variations due to the different characteristics of the food medium so we decided to use distilled water.

Another important parameter is the choice of antagonist material. Many authors prefer to use standardized bodies made of different types of ceramic materials in order to reduce the variations in the test results (4), (5), (6). We chose prefabricated ceramic spheres made of silicon nitride (Si3N4), which are also applied in machine engineering for the fabrication of ceramic rolling bearings. The spheres are standardized by ISO 3290. third edition. $2001 / 12 / 01$, the departure from roundness and the variation in radius being $0.13 \mu \mathrm{m}$ and the variation in the surface roughness - up to $0.014 \mu \mathrm{m}$. Vickers hardness according to the manufacturer is $24000 \mathrm{~N} / \mathrm{mm} 2$ which in a greater degree resembles the enamel's hardness compared to alumina and zirconia ceramics which were also considered as possibility for antagonist's material.

The in vitro wear testing results in the present paper are consistent with the results obtained in previous studies using the same chewing simulator, composite material and testing method (7), (8).

The main goal of every dental research in general is to be utilized by practitioners in their clinical work (9), (10) and to be a subject of further evaluation and comparison by other researchers. Therefore, the laboratory conditions should be properly clarified so that similar experiments could be run by scientists around the globe and realistic and verifiable results can be obtained.

\section{Conclusion}

The aim of the present study was to identify whether how much the antagonist rise and descend during an in vitro wear testing experiment can influence the results. This parameter is usually not listed in wear simulator settings and according to author' knowledge was never discussed as one that can influence the in vitro wear testing results. Within the limitation of our study we demonstrated that a statistically significant difference exists when this parameter is altered. Since it is not a material-related property but has the potential to influence data regarding the wear resistance of dental material we believe that this parameter should be mentioned, recorded and controlled once chewing simulators setting parameters in an in vitro wear testing are discussed.

\section{References}

1. Chakalov IE. Wear resistance of dental materials. In-vitro studies - PhD. Thesis. Sofia, 2014.

2. Kohyama, K., E. Hatakeyama, H. Dan and T. Sasaki. Effects of sample thickness on bite force for raw carrots and fish gels. - Journal of Texture Studies, 2005, 36, 157-173.

3. Le Gall, M. G., J. F. Lauret. Occlusion et function, une approche clinique rationnelle. - CdP Editions, 2002

4. Lasserre JF. Thèse pour le Doctorat d'Universite de Bordeaux2. Mention Sciences Biologiques et Médicales. Option Sciences Odontologique. Recherches sur l'usure dentaire et évaluation "in-vitro" de biomateriaux restaurateurs avec le simulateur d'usure UVSB2. 2003. 
5. Heintze SD: How to qualify and validate wear simulation devices and methods. Dent Mater. 2006 Aug; 22(8):712-34.

6. Kunzelmann, K.-H.: Verschleißanalyse und -quantifizierung von Füllungsmaterialien in vivo und in vitro. Shaker Verlag, Aachen 1998.

7. Chakalov I., Ivanova P., Apostolov N. Influence of different tribological mechanisms on in-vitro wear simulation results of composites. Medinform 2014, vol 1, Issue 1, 28-34

8. Chakalov I, Ivanova P. In vitro wear resistance of five composite restorative materials: A comparative research study. Journal of Lebanese Dental Association. Volume 52-No 1-JanuaryJune 2017; p47-53.

9. Dimova, M: Occlusion and articulation in bruxism and bruxomania investigated with the system Tscan, Journal of IMAB, 2014, 20,5,655-660.

10. Dimova, M.: Registration of centrc occlusion in patients with bruxism and bruxomania through articulating paper and the system T-scan-comparative analysis, Journal of IMAB,2014,20,520-525

\section{Corresponding author:}

Assistant Professor Ivan Chakalov,

Prosthetic Dentistry,

Faculty of Dental Medicine,

Medical University - Sofia

1 G. Sofiiski Str., 1431

Sofia, Bulgaria

email: uanio@yahoo.com 